\title{
Trigeminal Rhizotomy Performed with Modern Image-guided Linac: Case Report
}

Bobby C. Baker, Thomas A. Sullivan

1.

Corresponding author: Bobby C. Baker, bb@cancermd.net

\begin{abstract}
Introduction: There are substantive articles published within the last ten years describing the use of linear accelerator (Linac) based treatment of trigeminal neuralgia (TN). In this paper, we report a single case of a patient with classic TN successfully treated by radiosurgical rhizotomy using a modern image-guided linear accelerator with a high-definition multileaf collimator and an optically guided targeting system. The measurement of absolute dose prior to treatment greatly facilitated our confidence with radiosurgical lesioning.
\end{abstract}

Materials and Methods: This 54-year-old patient had a six-year history of lancinating pain involving the lower third of the right face which dramatically intensified in December of 2012, shortly after beginning Vincristine. A treatment plan was generated using a nine-field dynamic arc technique. A target volume was placed at 2-3 $\mathrm{mm}$ from the root entry zone measuring $6 \times 3 \times 3 \mathrm{~mm}$. The PTV dose was prescribed to $60 \mathrm{~Gy}$ to the $80 \%$ isodose line. Initial verification of absolute dose using Gafchromic film resulted in an increase of the planned MU’s by $7.5 \%$. Remeasurement improved the absolute dose to within $\pm 2 \%$ of the calculated dose.

Results: The patient was evaluated two weeks and three months following treatment. Her trigeminal pain was $80 \%$ resolved after two weeks and $100 \%$ at the three-month evaluation. She required none of her chronic pre-treatment medications and demonstrated no clinically detected sensory deficits.

Conclusions: Linear accelerator-based radiosurgery utilizing intrafraction motion management and image guidance offers an acceptable alternative for the radiation treatment of trigeminal neuralgia patients if absolute doses are measured prior to treatment.

Received 09/15/2013 Review began 09/15/2013 Published 09/15/2013

\section{() Copyright 2013}

Baker et al. This is an open access article distributed under the terms of the Creative Commons Attribution License CC-BY 3.0., which permits unrestricted use, distribution, and reproduction in any medium, provided the original author and source are credited.
Categories: Medical Physics, Radiation Oncology, Neurosurgery

Keywords: rhizotomy, stereotactic radiosurgery, multileaf collimator, trigeminal neuralgia, srs, linac, mlc

\section{Introduction}

The Gamma Knife has historically been the "Gold-Standard" for the radiosurgical lesioning of trigeminal neuralgia (TN) [1-2]. By administering 60-90 Gy with a Co-60 source through a $4 \mathrm{~mm}$ aperture, the Gamma Knife has been shown to produce conformal isodose distributions and correspondingly excellent clinical results [3-7].

Many radiation oncology facilities are limited to linear accelerator-based radiation treatments and must resort to referring TN patients to the nearest Gamma Knife or CyberKnife facilities. The advent of imageguided linear accelerators with intrafraction motion management systems have created an opportunity to safely treat these patients with the same level of accuracy and precision as framed-based and dedicated radiosurgery systems. This offers radiation oncologists the opportunity to offer this type of treatment in community-based practices.

There are substantive articles published within the last ten years describing the use of linear accelerator (linac)-based treatment of TN [8-12]. The consensus indicates that radiosurgery treatment of TN with a linear accelerator offers comparable outcomes to frame-based Gamma Knife systems. Most of these linac techniques use a 4 to $5 \mathrm{~mm}$ fixed circular cone to collimate the dose.

Pacific Cancer Institute of Maui recently commissioned a Varian Truebeam STx linear accelerator with an HD120 MLCs and AlignRT, an optically guided targeting system (Varian Medical Systems, Palo Alto, CA). Our premise for treating TN patients was based on the assumption that the $2.5 \mathrm{~mm}$ multi-leaf collimator (MLC) might provide sufficient resolution for trigeminal nerve lesioning, if one were to utilize pretreatment absolute dose confirmation. In this paper, we report a single case of a patient with classic trigeminal neuralgia successfully treated by radiosurgical rhizotomy using a modern image-guided linear accelerator.

\section{Case Presentation}

This 54-year-old patient had a six-year history of lancinating pain involving the lower third of the right face, 


\section{Cureus}

which dramatically intensified in December of 2012 shortly after beginning Vincristine. Despite maximum doses of amitriptyline, gabapentin, and narcotics, the patient was having no pain relief. Because the patient was actively receiving chemotherapy for an advanced nodular lymphoma, she was judged to be at high risk of a postoperative infection and a good candidate for microvascular decompression.

The trigeminal neuralgia patient was immobilized during computerized tomography (CT) simulation with a custom Accuform cushion and aquaplast mask with a built-in bite block. A CT was acquired with $1.25 \mathrm{~mm}$ slice thicknesses throughout the head. T1 and T2-weighted magnetic resonance imaging (MRI) images were reconstructed from volume acquisitions into $1 \mathrm{~mm}$ slices. These images were fused with the treatment planning CT dataset for identification of the trigeminal root entry zone (REZ). The planned target volume measured approximately $6 \times 3 \times 3 \mathrm{~mm}$. Critical organs at risk (OAR), such as the brainstem, cochlea, ipsilateral Gasserian ganglion, and the $7^{\text {th }} / 8^{\text {th }}$ nerve complex, were also delineated (Figure 1).

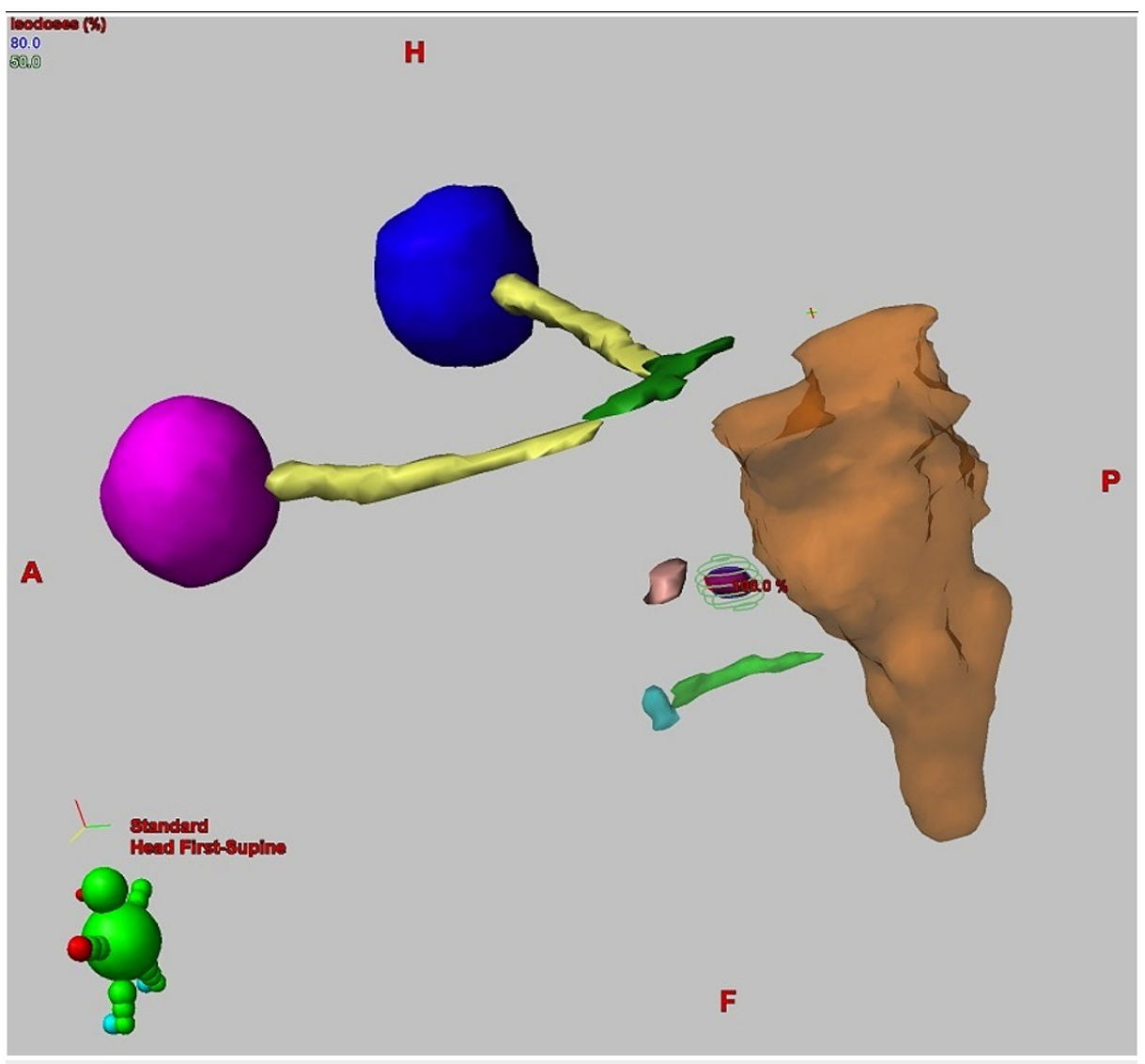

FIGURE 1: Relevant Contours

Involved anatomy demonstrating the brainstem (orange), left cochlea (cyan), the Gasserian ganglion (pink), the 7 th/8th nerve complex (It. green), the eyeballs, optic nerves, optic chiasm, and the trigeminal nerve PTV within the $80 \%$ and $50 \%$ isodose clouds.

The Eclipse treatment planning computer, V11.0 (Varian Medical Systems, Palo Alto, CA), was used to calculate the isodose distribution using nine dynamic arcs strategically placed to minimize the dose to the OARs (Figure 2). Several of the arcs were repeated (reversed direction) to achieve the MU/degree within machine tolerances $(\leqslant 60)$. VMAT (Volumetric Modulated Arc Therapy) was not used because the small beamlets produced by the MLC modulation were thought to add more uncertainty to the accuracy of dose. The prescribed dose to the PTV was 60 Gy in one fraction to the $80 \%$ isodose line, covering $95 \%$ of the delineated target with a Dmax of 75 Gy. Each arc used a photon energy of 6XFFF (flattening filter free), 1400 cGy/min dose rate, a $1 \times 1 \mathrm{~cm}^{2}$ collimator jaw setting, and the HD120 MLCs for "beams-eye" conformal shaping to the target volume. Using the anisotropic analytical algorithm (AAA), the resultant plan confirmed acceptable conformality for treating the TN patient. 


\section{Cureus}

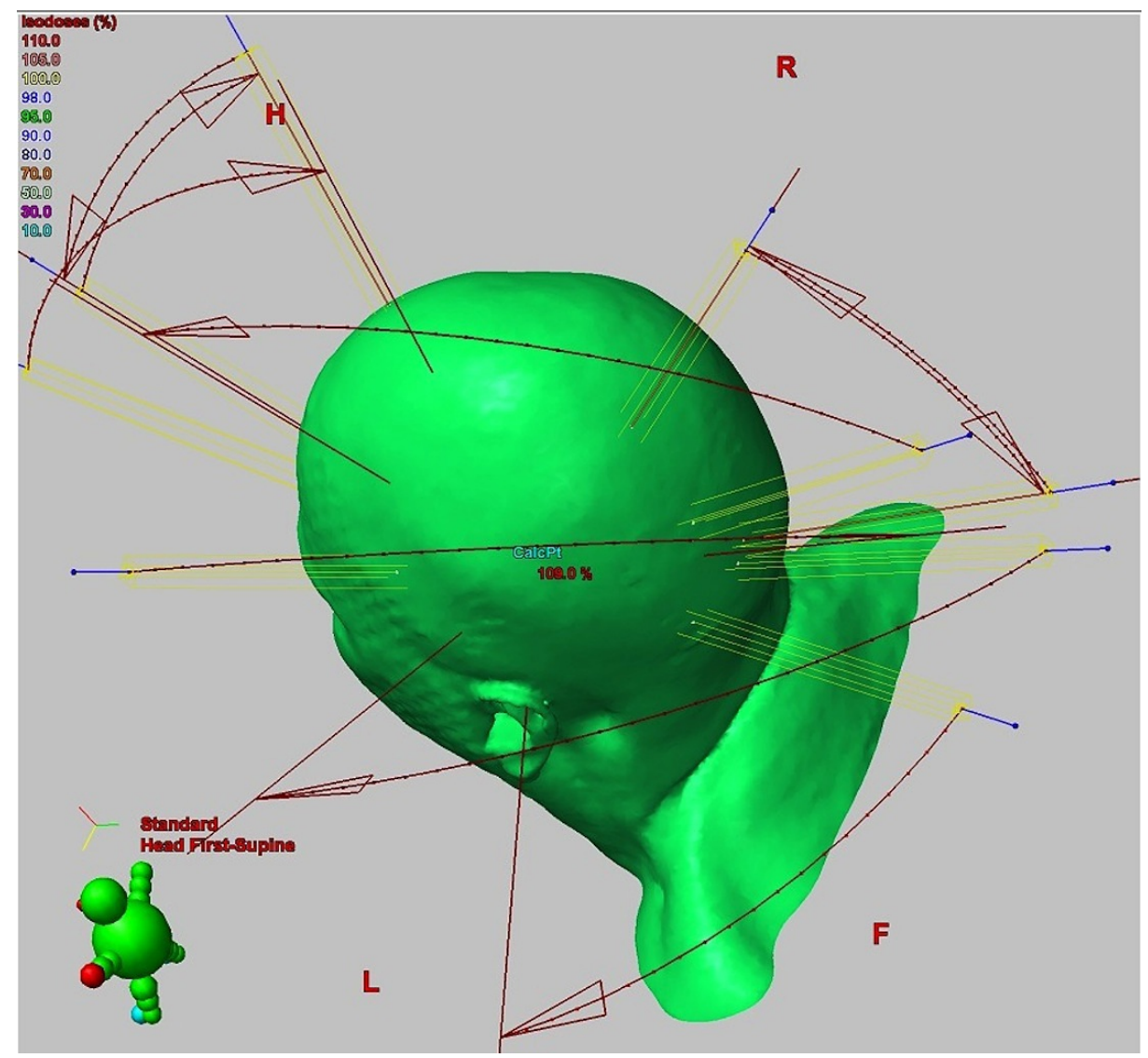

FIGURE 2: Dynamic Arc Configuration

Representative dynamic arcs for treatment of trigeminal neuralgia.

MD-V3 Gafchromic film [13] was used for verification QA of the treatment plan. The extended dynamic dose range of this dosimetry media (1 to $100 \mathrm{~Gy}$ ) and its high spatial resolution (down to $5 \mu \mathrm{m}$ ) makes it the ideal dosimeter to verify the absolute dose and distribution for such a small volume. A verification plan on a solid water phantom was created from the Eclipse treatment plan and sent to the TrueBeam STx for quality assurance measurements on the MD-V3 film. A separate set of dose calibration films were also acquired, and an H\&D curve was obtained using calibrated ionization chamber measurements. The Gafchromic film was scanned on a flatbed scanner and analyzed using DoseLab software [14]. The results of the relative dose comparison showed that the measured isodose distribution was very close to the predicted (Figure 3).

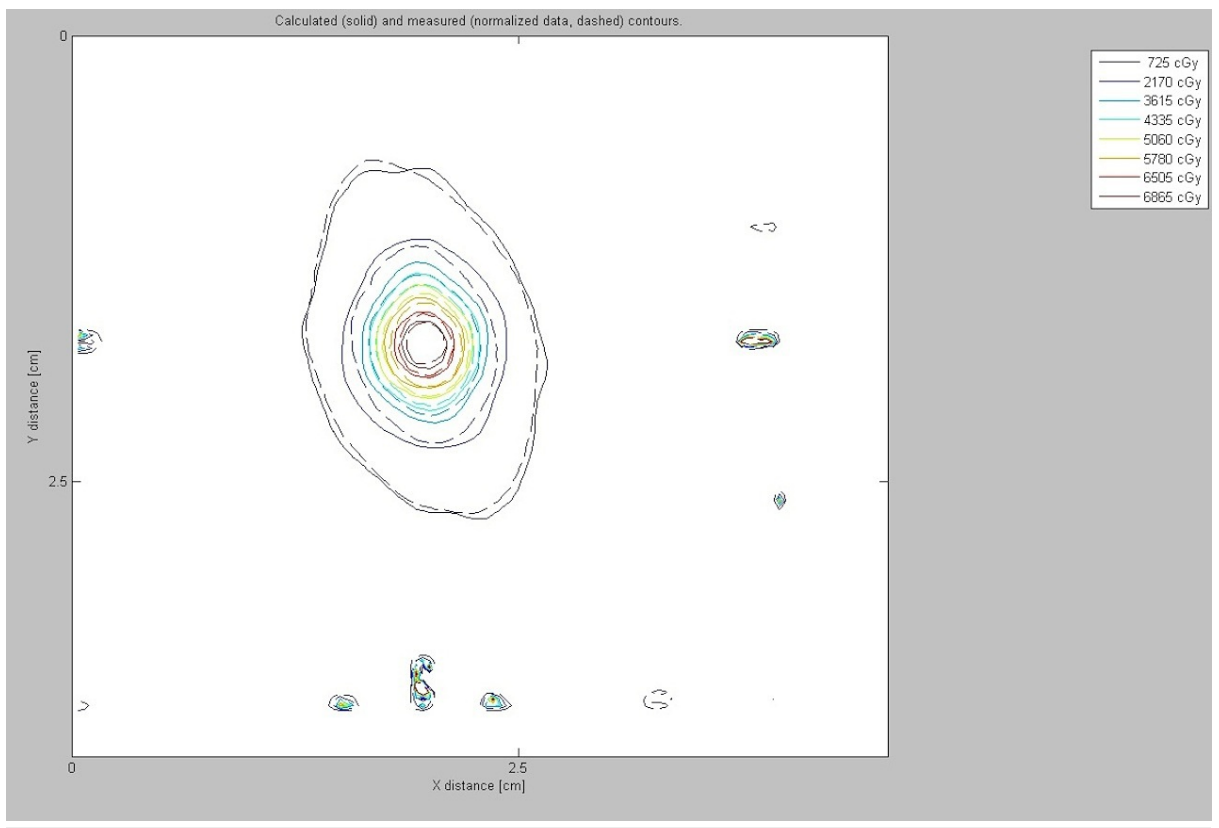




\section{Cureus}

\section{FIGURE 3: Isodose Comparison}

Comparison between measured (dashed) and calculated (solid) isodose lines. (Note: contours at edges were from marker used for isocenter localization.)

The gamma factor, using 3\% dose and $1.0 \mathrm{~mm}$ distance to agreement (DTA), averaged a $99.5 \%$ pass rate over four separate repeat measurements. The gamma values showed excellent results (Figure 4).

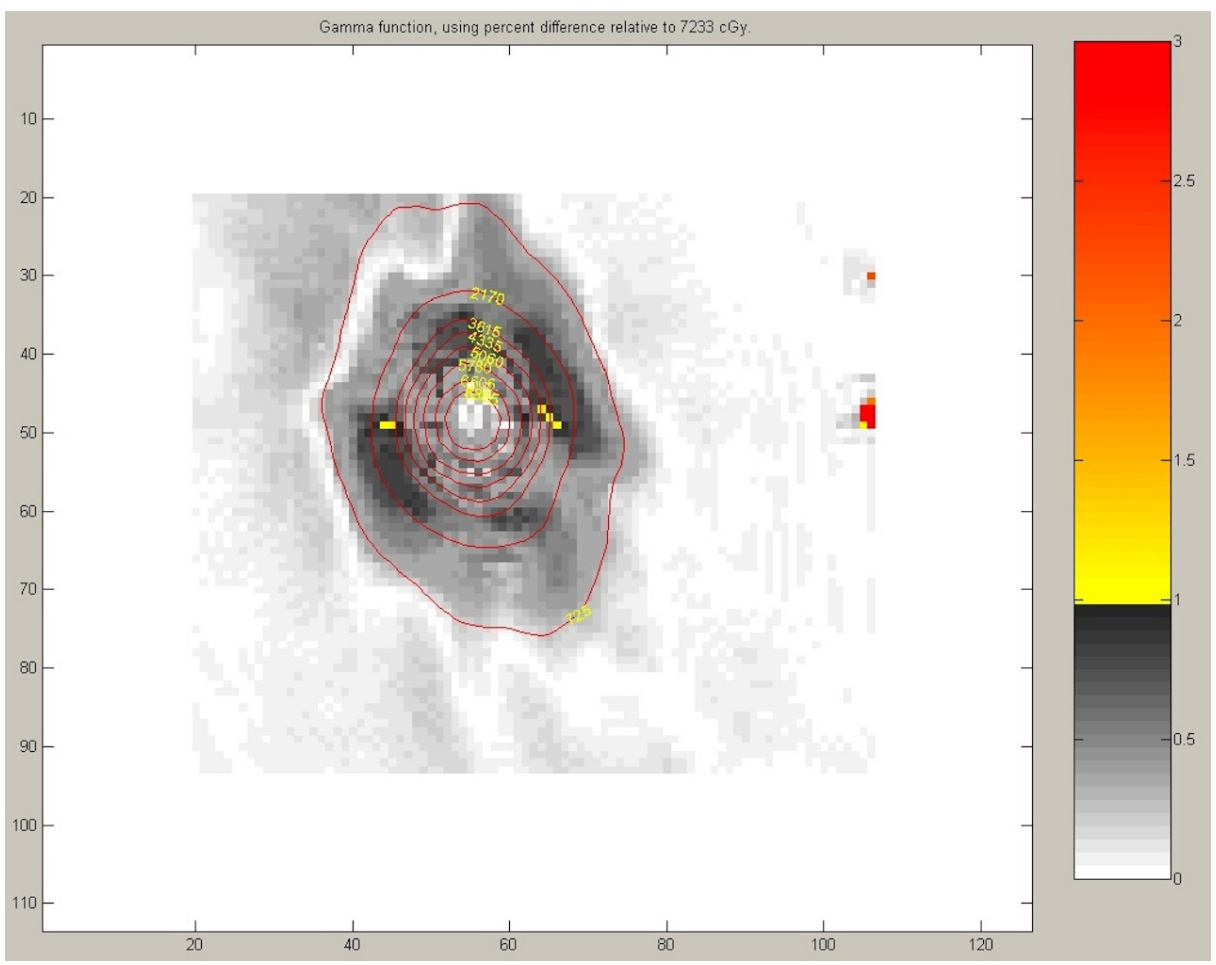

\section{FIGURE 4: Gamma Factors}

Gamma factors for trigeminal QA plan using 3\% dose/1 mm DTA. (Pixel values $\geq 1$ in color, lower acceptable values in B/W.)

Even though the measured relative isodose distribution was well-predicted by the treatment planning software, the absolute dose was measured at between 7-9\% less than the calculated values. Since the MLCs were not used for dose modulation in a dynamic arc treatment (the plan was not VMAT), a multiplicative factor could be used to correct the plan monitor units (MUs) and thus, the final dose delivered. The measured absolute dose was improved to $\pm 2 \%$ of the calculated absolute dose by increasing the plan MUs by $7.5 \%$. The resultant measured isodose distribution confirmed the same good agreement with the original plan isodose distribution. The gamma factor still exceeded a $99 \%$ pass rate.

After acceptable dose quality assurance was obtained, the focus was switched to accurate patient setup and immobilization. The aquaplast mask was cut out around the forehead, eyes, and nose area so that the patient could be monitored during treatment with the AlignRT [15] surface rendering module (Figure 5). This real time 3D system uses cameras and infra-red light to monitor the patient's skin surface with submillimeter accuracy [16-19]. 


\section{Cureus}

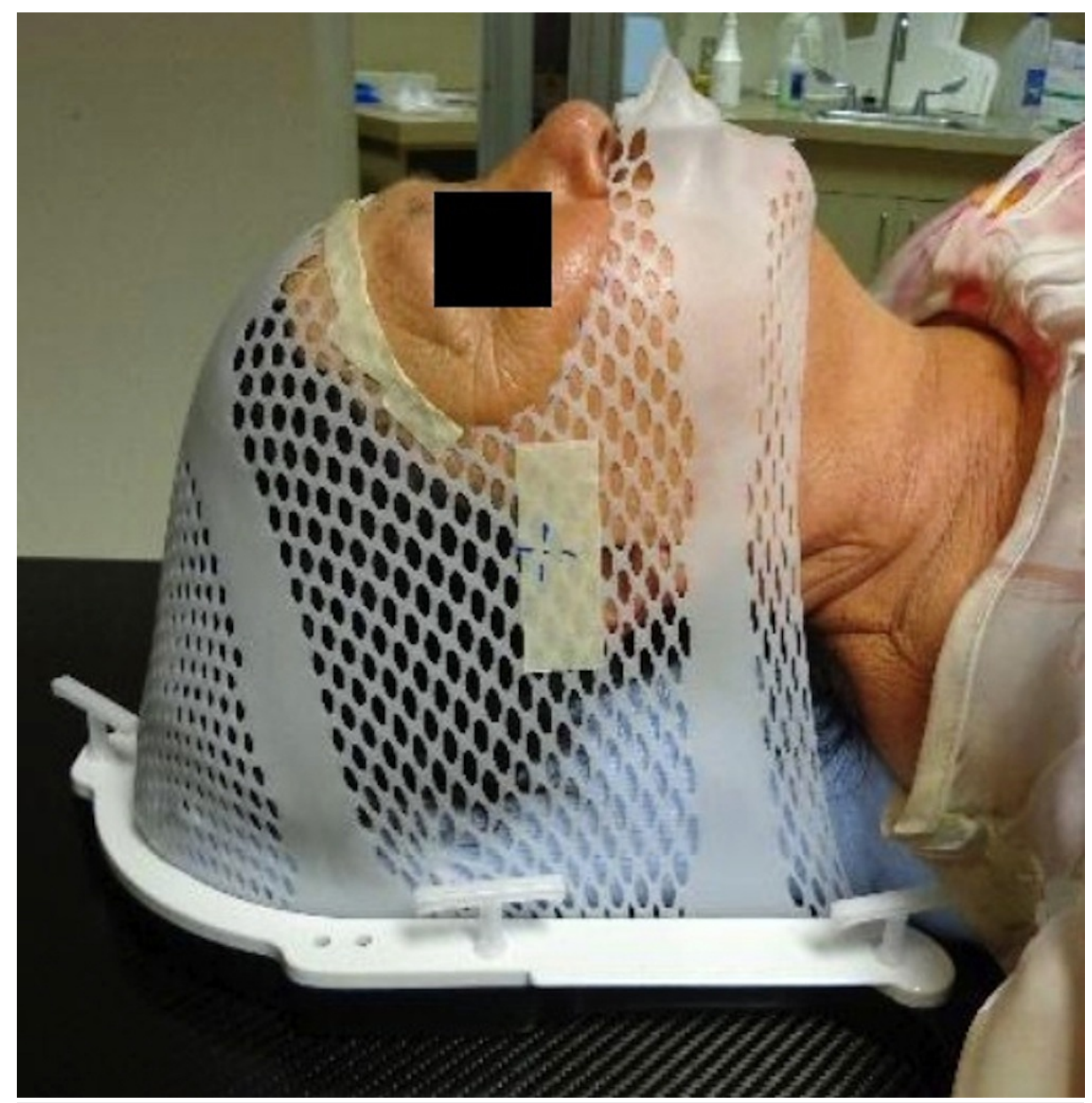

FIGURE 5: Custom Aquaplast Mask

Aquaplast mask with facial feature cutout and bite block placement.

Initially, the patient was set up with the AlignRT reference surface from the body structure reconstructed from the simulation CT dataset. A cone beam CT (CBCT) was acquired, and the necessary table shifts were made. If any of the rotational shifts were greater than $1.5^{\circ}$, the patient's head was gently rotated and the CBCT was repeated. After the appropriate isocenter was obtained, a new reference surface was acquired and monitored during the treatment. The patient's isocenter maintained a vector accuracy of $1 \mathrm{~mm}$ during the entire treatment.

\section{Clinical results}

The patient was clinically evaluated at two weeks and three months following treatment. Her trigeminal pain was $80 \%$ resolved after two weeks, and $100 \%$ at the three-month evaluation. She was completely titrated off of all of her pre-treatment medications. She experienced no treatment-related

sequelae. Specifically, the sensory function of all three trigeminal divisions was subjectively, and upon clinical testing, without deficit.

\section{Discussion}

This treatment utilized a new, modern image-guided linear accelerator with high-definition MLC's to replace cone-based SRS delivery. Furthermore, CBCT and real-time surface monitoring was used instead of a framebased system. The equipment for this technique is becoming more and more prevalent in community-based radiation oncology clinics.

The method utilized for treating our patient was relatively conventional, except for the treatment planning and the even more critical absolute dosimetry measurements. Since plans can be varied dramatically with respect to the number, length, and placement of arcs, the first two of these parameters were determined primarily by minimizing their interception with any organs at risk (OAR). The total number of arcs was dictated by the MU/degree capabilities of the linear accelerator and the need to maintain any normal tissue to a low integral dose. 
We concluded that a standardized immobilization position during the simulation process would help alleviate the necessity to recreate completely different arc series for each new patient. Dynamic arcs were chosen as the delivery technique over static IMRT fields or RapidArc (VMAT) for a number of reasons. The total number of static fields needed to obtain an acceptable plan would be time-prohibitive (CyberKnife plans are typically 80-120 beam angles) and would substantially increase the total delivery time on a gantrybased linac system (gantry/couch rotation). As stated earlier, VMAT was not used because the small beamlets produced by the MLC modulation would add more uncertainty to the accuracy of the dose. Also, the absolute dose could not be adjusted accurately by simply multiplying the MUs from a VMAT plan as with a dynamic arc plan. The total treatment time was about one hour (CBCT setup time of 45 min and 12-13 minutes of beam-on time).

Even though the results are preliminary, they are very encouraging. We believe that the advent of this technique will provide a useful treatment option for radiation oncologists who have access to the equipment types that have been described in this paper. Our team spent countless hours perfecting this technique prior to its execution, and it is our hope that other professionals will be encouraged to consider our method as a treatment option when absolute dose can be confidently confirmed prior to treatment.

\section{Conclusions}

The TrueBeam STx linear accelerator with HD120 MLC's, combined with the AlignRT intrafraction motion management system and CBCT image guidance, offers an acceptable alternative for the radiation treatment of TN patients when compared to frame- based linear accelerator and Co-60 systems. The HD 120 MLC's can accurately replace cones for the necessary enhanced collimation when the absolute doses have been measured prior to treatment. The end-to-end quality assurance measurements provided the confidence needed to safely and accurately deliver high dose to the trigeminal nerve [20].

Further immobilization, treatment planning (i.e. number of arcs, angles and arc degrees), and absolute dose verification techniques should be explored and reported.

\section{Additional Information \\ Disclosures}

Human subjects: Consent was obtained by all participants in this study. Conflicts of interest: In compliance with the ICMJE uniform disclosure form, all authors declare the following: Payment/services info: All authors have declared that no financial support was received from any organization for the submitted work. Financial relationships: All authors have declared that they have no financial relationships at present or within the previous three years with any organizations that might have an interest in the submitted work. Other relationships: All authors have declared that there are no other relationships or activities that could appear to have influenced the submitted work.

\section{References}

1. Kondziolka D, Lunsford Kondziolka D, Lunsford D, Flickinger JC, Young RF, Vermeulen S, Duma CM: Stereotatic radiosurgery for trigeminal neuralgia: A multi-institutional study using the gamma unit . J Neurosurg. 1996, 84:940-945.

2. Kondziolka D, Perez B, Flickinger JC, Habeck M, Lunsford LD: Gamma Knife radiosurgery for trigeminal neuralgia: Results and expectation. Arch Neurol. 1998, 55:1524-1529.

3. Brisman R: Gamma Knife radiosurgery for primary management for trigeminal neuralgia . J Neurosurg. 2000, 93:159-161.

4. McNatt SA, Yu C, Giannotta SL, Zee CS, Apuzzo ML, Petrovich Z: Gamma Knife radiosurgery for trigeminal neuralgia: Results and expectation. Neurosurg. 2005, 56:1295-1303.

5. Adler, JR Jr, Bower R, Gupta G, Lim M, Efron A, Gibbs IC, Chang SD, Soltys SG: Non-isocentric radiosurgical rhizotomy for trigeminal neuralgia. Neurosurg. 2009, 64:A84-90.

6. Sheehan J, Pan HC, Stroila M, Steiner L: Gamma Knife surgery for trigeminal neuralgia: outcomes and prognostic factors. J Neurosurg. 2005, 102:434-441.

7. Kondziolka D, Zorro O, Lobato-Polo J, Kano H, Flannery TJ, Flickinger JC, Lunsford LD: Gamma Knife stereotactic radiosurgery for idiopathic trigeminal neuralgia. J Neurosurg . 2010, 112:758-765.

8. Chen JC, Girvigian M, Greathouse H, Miller M, Rahimian J: Treatment of trigeminal neuralgia with linear accelerator radiosurgery: Initial results. J Neurosurg. 2004, 101:346-350.

9. Gerbi BJ, Higgins PD, Cho KH, Hall WA: Linac-based stereotactic radiosurgery for treatment of trigeminal neuralgia. J Appl Clin Med Phys. 2004, 5:80-92.

10. Ma L, Kwok Y, Chin LS, Yu C, Regine WF: Comparative analyses of linac and Gamma Knife radiosurgery for trigeminal neuralgia treatments. Phy Med Biol. 2005, 50:5217-5227.

11. Hazard LJ, Wang B, Skidmore TB, Chern SS, Salter BJ, Jensen RL, Shrieve DC: Conformity of Linac-based stereotactic radiosurgery using dynamic conformal arcs and micro-multileaf collimator. Int J Radiat Oncol Biol Phys. 2009, 73:562-570.

12. Frighetto L, De Salles AA, Smith ZA, Goss B, Selch M, Solberg T: Noninvasive linear accelerator radiosurgery as the primary treatment for trigeminal neuralgia. Neurol. 2004, 62:660-662.

13. International Specialty Products. (2013). Accessed: 10/1/13: http://medicalphysicsweb.org/cws/company/C000003429.

14. Mobius Medical Systems. (2013). Accessed: 10/1/13: http://www.mobiusmed.com/. 


\section{Cureus}

15. VisionRT. (2013). Accessed: 10/1/13: http://www.visionrt.com/.

16. Peng, JL, Kahler, D, Li, JG, et al.: Characterization of a real-time surface image-guided stereotactic positioning system. Med. Physics . 2010, 37:5421-5433.

17. Li G, Ballangrud A, Cheng Kuo L, et al.: Motion monitoring for cranial frameless stereotactic radiosurgery using video-based three-dimensional optical surface imaging. Med. Physics . 2011, 38:3981-3994.

18. Cervino LI, Pawlicki T, Lawson JD, Jiang SB: Frame-less and mask-less cranial stereotactic radiosurgery: A feasibility study. Phys Med Biol. 2010, 55:1863-1873.

19. Cervino LI, Detorie N, Taylor M, et al.: Initial clinical experience with a frameless and maskless stereotactic radiosurgery treatment. Pract Radiat Oncol. 2012, 2:54-62.

20. Bogdanich W, Rebelo K: A pinpoint beam strays invisibly, harming instead of healing . NY Times. Dec 29, 2010 , 